\title{
HOMOLOGY AND THE KOSZUL COMPLEX
}

\author{
by D. J. MOORE
}

(Received 15 October, 1969)

Introduction. Let $R$ be a commutative ring with an identity element, $E$ a (unitary) $R$-module, and $x_{1}, x_{2}, \ldots, x_{s}$ elements of $R$. In these circumstances it is possible to form the Koszul complex $\dagger K\left(x_{1}, x_{2}, \ldots, x_{s} \mid E\right)$ of $E$ with respect to $x_{1}, x_{2}, \ldots, x_{s}$ and to investigate the implications, for $E$ and $x_{1}, x_{2}, \ldots, x_{s}$, if certain of the homology modules of this complex vanish. This was first undertaken by M. Auslander and D. A. Buchsbaum [1]. Among the many results they obtain, the following [1, Proposition 2.8, p. 632] is of particular interest in connection with the present paper:

If $R$ is Noetherian, $E$ is finitely generated, and $x_{1}, x_{2}, \ldots, x_{s}$ belong to the Jacobson radical of $R$, then the statements

(a) $x_{1}, x_{2}, \ldots, x_{s}$ is an $R$-sequence on $E$,

(b) $H_{p} K\left(x_{1}, x_{2}, \ldots, x_{s} \mid E\right)=0$ for all $p>0$,

(c) $H_{1} K\left(x_{1}, x_{2}, \ldots, x_{s} \mid E\right)=0$,

are all equivalent.

Here and in the sequel $H_{\mu} K\left(x_{1}, x_{2}, \ldots, x_{s} \mid E\right)$ denotes the $\mu$ th homology module of $K\left(x_{1}, x_{2}, \ldots, x_{s} \mid E\right)$.

Now it is evident that what causes the homology modules of the complex to vanish is the way the elements $x_{i}$ act on $E$, and the various general assumptions placed on $R, E$ and the $x_{i}$ serve to ensure that this action has suitable general characteristics. However these overall assumptions are, in fact, unnecessarily strong for this purpose and in the present paper the question of suitable interaction is dealt with more directly. For example, it emerges that it is sufficient that $E$ should be normal with respect to $x_{1}, x_{2}, \ldots, x_{s}$. This means that if $E^{\prime}$ is any submodule of $E$, then

$$
\left(x_{1}, x_{2}, \ldots, x_{s}\right)^{m} E \cap E^{\prime} \subseteq\left(x_{1}, x_{2}, \ldots, x_{s}\right) E^{\prime},
$$

provided that $m$ is large enough. Again, if we weaken the general assumptions concerning $R, E$ and $x_{1}, x_{2}, \ldots, x_{s}$, then (a) is unnecessarily restrictive to ensure (b). For this situation the following adjustment is appropriate. First we say that $E$ is trivial with respect to $x_{1}, x_{2}, \ldots, x_{s}$ if it is normal with respect to these elements and $E=\left(x_{1}, x_{2}, \ldots, x_{s}\right) E$. We then describe $x_{1}, x_{2}, \ldots, x_{s}$ as a regular sequence $\ddagger$ on $E$ if, for each $i(1 \leqq i \leqq s)$, the factor module

$$
\frac{\left(x_{1}, \ldots, x_{i+1}\right) E:_{E} x_{i}}{\left(x_{1}, \ldots, x_{i-1}\right) E}
$$

is trivial with respect to $x_{1}, x_{2}, \ldots, x_{s}$. With these modifications the result quoted above may now be replaced (see Theorems 1 and 2) by:

+ An elementary account of the Koszul complex will be found in [3, Chapter 8].

$¥$ In the case of an $\left(x_{1}, \ldots, x_{s}\right)$-normal module the order of the $x_{l}$ proves to be irrelevant. See Theorem 3. 
Let $E$ be normal with respect to $x_{1}, x_{2}, \ldots, x_{s}$. Then the statements

(a) $x_{1}, x_{2}, \ldots, x_{s}$ is a regular sequence on $E$,

(b) $H_{p} K\left(x_{1}, x_{2}, \ldots, x_{s} \mid E\right)=0$ for all $p>0$,

(c) $H_{1} K\left(x_{1}, x_{2}, \ldots, x_{s} \mid E\right)=0$,

are all equivalent.

In fact with this kind of adjustment the homology theory of the Koszul complex can be recast in a form which is readily developed within the framework of a general abelian category. This is what is done here. One advantage of this is that we obtain the dual theory as a bonus. Even in the case of Noetherian modules we get something extra. Thus the rigidity of the Koszul complex [1, Proposition 2.4, p. 632] is established in the quasi-commutative case and here the original arguments of Auslander and Buchsbaum are not directly applicable.

Some ideas used in this paper have their origins in [2]. I should like to thank Professor D. G. Northcott for the suggestions he has made during the preparation of the present paper.

1. Normality and triviality. Throughout this paper $\mathfrak{A}$ denotes a fixed abelian category and $\mathscr{F}$ the class of natural transformations of the identity functor. Suppose that $\omega \in \mathscr{F}$ and let $A$ be an $\mathfrak{Y}$-object. Then $\omega$ determines a morphism $\omega^{A}: A \rightarrow A$ which is such that if $f: A \rightarrow B$ in $\mathfrak{Y}$, then the diagram

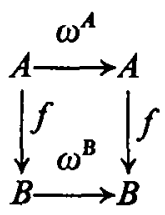

is commutative. The members of can be added and multiplied and, with respect to these operations, $\mathscr{F}$ resembles a ring. Indeed multiplication is commutative. There is also an identity element 1 with the property that, for each $A \in \mathfrak{U}, 1^{A}$ is the identity morphism of $A$. The image of the morphism $\omega^{A}$ will be denoted by $\omega A$. Thus $\omega A$ is a subobject of $A$. It is easily verified that if $\omega^{\prime}$ also belongs to $\mathscr{F}$, then

$$
\omega^{\prime}(\omega A)=\left(\omega^{\prime} \omega\right) A=\omega\left(\omega^{\prime} A\right) .
$$

Let $\omega_{1}, \omega_{2}, \ldots, \omega_{s}$ be a sequence†, $\Omega$ say, of members of $\mathscr{F}$. We put

$$
\Omega A=\omega_{1} A+\omega_{2} A+\ldots+\omega_{s} A
$$

this being regarded as a subobject of $A$. Note that if $f: A \rightarrow B$ in $\mathfrak{F}$, then

$$
f(\Omega A)=\Omega f(A) \text {. }
$$

Next let $A_{1}, A_{2}$ be subobjects of an object $C$. Then $\Omega A_{1}, \Omega A_{2}$ may also be regarded as subobjects of $C$. If now $A_{1} \leqq A_{2}$ in the partial preordering of the subobjects of $C$, then $\Omega A_{1} \leqq \Omega A_{2}$ as well.

t It is not until Section 2 that the order of the terms in the sequence has any importance. Note that there may be repetitions. 
Let us suppose that $\Omega$ and $\Omega^{\prime}$ denote the sequences $\omega_{1}, \omega_{2}, \ldots, \omega_{s}$ and $\omega_{1}^{\prime}, \omega_{2}^{\prime}, \ldots, \omega_{t}^{\prime}$ respectively. Then by (1.1),

$$
\Omega\left(\Omega^{\prime} A\right)=\Omega^{\prime}(\Omega A)=\left(\Omega \Omega^{\prime}\right) A,
$$

where by $\Omega \Omega^{\prime}$ we mean the products $\omega_{i} \omega_{j}^{\prime}$ arranged sequentially. Again if $p>0$ is an integer, we use $\Omega^{p}$ for the set of power products $\omega_{1}^{v_{1}} \omega_{2}^{v_{2}} \ldots \omega_{s}^{v_{s}}$, where $v_{1}+v_{2}+\ldots+v_{s}=p$, arranged as a sequence. This symbol satisfies

and

$$
\begin{aligned}
\Omega^{p} A & \leqq \Omega^{p-1} A, \\
\Omega^{p}\left(\Omega^{q} A\right) & =\Omega^{p+q} A,
\end{aligned}
$$

$$
\left(\Omega^{p}\right)^{q} A=\Omega^{p q} A \text {. }
$$

These preliminaries enable us to make the

Definition. The $\mathfrak{A}$-object $A$ is said to be " $\Omega$-normal" if, for any subobject $B$ of $A$, there exists a positive integer $p$, dependent upon $B$, such that

$$
\Omega^{p} A \cap B \leqq \Omega B,
$$

where these are considered as subobjects of $A$.

To illustrate this concept we prove

Proposition 1. Let $R$ be a ring with identity element and let $E$ be a Noetherian $R$-module. Then $E$ is normal with respect to any finite sequence of central elements of $R$.

Proof. Let $\Gamma$ denote the central ideal of $R$ generated by the central elements $\gamma_{1}, \gamma_{2}, \ldots, \gamma_{t}$. Let $K$ denote any submodule of $E$. Then, by the Artin-Rees Theorem [3, Theorem 1, p. 292], there exists an integer $q \geqq 0$ such that

$$
\Gamma^{n} E \cap K=\Gamma^{n-q}\left(\Gamma^{q} E \cap K\right)
$$

for all $n \geqq q$. Taking $n=q+1$, and noting that $\Gamma\left(\Gamma^{q} E \cap K\right) \subseteq \Gamma K$, we find that

$$
\Gamma^{n} E \cap K \subseteq \Gamma K
$$

We return now to the general situation in order to establish the basic facts concerning $\Omega$-normality.

LEMMA 1. Let $A$ be an $\mathfrak{O}$-object and let $r$ be a positive integer. Then $A$ is $\Omega$-normal when and only when $A$ is $\Omega^{r}$-normal.

Proof. Assume that $A$ is $\Omega$-normal, and let $B$ be a subobject of $A$. Let $0 \leqq m<r$. Then $\Omega^{m} B$ is a subobject of $A$, and hence there exists an integer $p_{m}$ such that

$$
\Omega^{p_{m}} A \cap \Omega^{m} B \leqq \Omega\left(\Omega^{m} B\right)=\Omega^{m+1} B .
$$

If $n=\max \left\{p_{0}, p_{1}, \ldots, p_{r-1}\right\}$, then

$$
\Omega^{n} A \cap B \leqq \Omega^{r} B
$$


If now we choose $t$ such that $r t \geqq n$, then

$$
\left(\Omega^{r}\right)^{t} A \cap B \leqq \Omega^{r} B
$$

Thus $A$ is $\Omega^{r}$-normal.

The converse assertion follows immediately since $\left(\Omega^{r}\right)^{q} A=\Omega^{r q} A$ (see (1.7)) and $\Omega^{r} A=\Omega\left(\Omega^{r-1} A\right) \leqq \Omega A$ (see (1.6)) as subobjects of $A$.

Lemma 2. Let $A$ be $\Omega$-normal. Then the subobjects and factor objects of $A$ are also

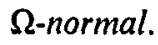

Proof. Let $B$ be any subobject of $A$ and let $B^{\prime}$ be a subobject of $B$. Since $B^{\prime}$ may be regarded as a subobject of $A$, there exists a positive integer $p$ such that

$$
\Omega^{p} A \cap B^{\prime} \leqq \Omega B^{\prime}
$$

Hence

$$
\Omega^{p} B \cap B^{\prime} \leqq \Omega^{p} A \cap B^{\prime} \leqq \Omega B^{\prime}
$$

and so $B$ is $\Omega$-normal.

Now let $C$ be an arbitrary factor object of $A$, where $f: A \rightarrow C$ denotes the canonical epimorphism. Let $A^{\prime}=\operatorname{Ker} f$, and let $N$ denote any subobject of $C$. Then $f^{-1}(N)$ is a subobject of $A$, and

$$
A^{\prime} \leqq f^{-1}(N)
$$

Since $A$ is $\Omega$-normal, there exists a positive integer $n$ such that

$$
\Omega^{n} A \cap f^{-1}(N) \leqq \Omega\left(f^{-1}(N)\right) .
$$

Since, by the modular law,

$$
\begin{aligned}
\left(\Omega^{n} A+A^{\prime}\right) \cap f^{-1}(N) & =\left(\Omega^{n} A \cap f^{-1}(N)\right)+A^{\prime} \\
& \leqq \Omega\left(f^{-1}(N)\right)+A^{\prime},
\end{aligned}
$$

we have the following ascending sequence of subobjects of $A$ :

$$
A^{\prime} \leqq\left(\Omega^{n} A+A^{\prime}\right) \cap f^{-1}(N) \leqq A^{\prime}+\Omega\left(f^{-1}(N)\right) \leqq A .
$$

But, on applying the epimorphism $f,(1.8)$ yields the ascending sequence

$$
0 \leqq \Omega^{n} C \cap N \leqq \Omega N \leqq C
$$

of subobjects of $C$. Thus $C$ is $\Omega$-normal.

LEMMA 3. Let $A_{1}, A_{2}, \ldots, A_{n}$ be $\Omega$-normal objects of $\mathfrak{A}$. Then their biproduct $A_{1} * A_{2} * \ldots * A_{n}$ is also $\Omega$-normal.

Proof. It is sufficient to consider the case $n=2$. Let $A=A_{1} * A_{2}$ and denote the canonical embedding $A_{i} \rightarrow A$ and projection $A \rightarrow A_{i}$ by $\sigma_{i}$ and $\pi_{i}$ respectively $(i=1,2)$. Let $D$ be a subobject of $A$, and write $D_{1}=\sigma_{1}^{-1}(D), D_{2}=\pi_{2}(D)$.

c 
Since $A_{1}$ is $\Omega$-normal, there exists a positive integer $p_{1}$ such that

$$
\Omega^{p_{1}} A_{1} \cap D_{1} \leqq \Omega D_{1} .
$$

Since $A_{2}$ is $\Omega$-normal, it is also $\Omega^{p_{1}}$-normal, and hence there exists a positive integer $p_{2}$ such that

$$
\left(\Omega^{p_{1}}\right)^{p_{2}} A_{2} \cap D_{2} \leqq \Omega^{p_{1}} D_{2} .
$$

Set $p=p_{1} p_{2}$. We claim that

Now

$$
\Omega^{p} A \cap D \leqq \Omega D .
$$

$$
\begin{aligned}
\Omega^{p} A \cap D & \leqq \pi_{2}^{-1}\left(\pi_{2}\left(\Omega^{p} A \cap D\right)\right) \leqq \pi_{2}^{-1}\left(\Omega^{p} A_{2} \cap D_{2}\right) \\
& \leqq \pi_{2}^{-1}\left(\Omega^{p_{1}} D_{2}\right) \quad(\text { by }(1.10)) \\
& =\pi_{2}^{-1}\left(\pi_{2}\left(\Omega^{p_{1}} D\right)\right)=\Omega^{p_{1}} D+A_{1},
\end{aligned}
$$

and therefore

$$
\begin{aligned}
\left(\Omega^{p} A \cap D\right) \cap\left(\Omega^{p_{1}} A\right) & \leqq\left(\Omega^{p_{1}} D+A_{1}\right) \cap\left(\Omega^{p_{1}} A\right) \\
& =\Omega^{p_{1}} D+\left(A_{1} \cap \Omega^{p_{1}} A\right) \\
& =\Omega^{p_{1}} D+\Omega^{p_{1}} A_{1},
\end{aligned}
$$

since

$$
\Omega^{p_{1}} A \cap A_{1}=\Omega^{p_{1}} A_{1}
$$

Hence

Thus

$$
\begin{aligned}
\left(\Omega^{p} A \cap D\right) \cap D & \leqq\left(\Omega^{p_{1}} D+\Omega^{p_{1}} A_{1}\right) \cap D=\Omega^{p_{1}} D+\left(\Omega^{p_{1}} A_{1} \cap D\right) \\
& \leqq \Omega^{p_{1}} D+\Omega D_{1} \quad(\text { by }(1.9)) \\
& \leqq \Omega D .
\end{aligned}
$$

$$
\Omega^{p} A \cap D \leqq \Omega D,
$$

and so $A$ is $\Omega$-normal, as required.

To supplement the notion of $\Omega$-normality we need the concept of $\Omega$-triviality. This is defined as follows.

Definition. The $\mathfrak{Q}$-object $A$ is said to be " $\Omega$-trivial " if $A$ is $\Omega$-normal and $A=\Omega A$.

We note that if $A$ is an $\Omega$-trivial $\mathfrak{A}$-object, then $A$ is $\Omega^{p}$-trivial for every positive integer $p$.

LEMMA 4. Let

$$
0 \rightarrow A \stackrel{f}{\rightarrow} B \stackrel{g}{\rightarrow} C \rightarrow 0
$$

be an exact sequence of $\Omega$-normal $\mathfrak{M}$-objects. Then $B$ is $\Omega$-trivial if and only if both $A$ and $C$ are $\Omega$-trivial. 
Proof. Assume that $B$ is $\Omega$-trivial. Then

$$
C=g(B)=g(\Omega B)=\Omega(g(B))=\Omega C .
$$

Hence $C$ is $\Omega$-trivial. Since $B$ is $\Omega$-normal, and $A$ is a subobject of $B$, there exists an integer $n$ such that

$$
\Omega^{n} B \cap A \leqq \Omega A \text {. }
$$

However $B=\Omega^{n} B$, and so $A \leqq \Omega A$. It thus follows that $A$ is also $\Omega$-trivial.

Conversely, assume that $A$ and $C$ are both $\Omega$-trivial. Then

$$
g^{-1}(g(\Omega B))=g^{-1}(\Omega(g(B)))=g^{-1}(\Omega C)=g^{-1}(C)=g^{-1}(g(B)) .
$$

Thus

Again,

$$
\Omega B+\operatorname{Ker} g=B+\operatorname{Ker} g
$$

Hence

$$
f\left(f^{-1}(B)\right)=f(A)=f(\Omega A)=f\left(\Omega\left(f^{-1}(B)\right)\right) \leqq f\left(f^{-1}(\Omega B)\right)
$$

$B \cap \operatorname{Im} f \leqq \Omega B \cap \operatorname{Im} f$.

Thus

$$
B \cap \operatorname{Im} f=\Omega B \cap \operatorname{Im} f .
$$

Since $\Omega B \leqq B$, and since $\operatorname{Im} f=\operatorname{Ker} g$, it follows that $B=\Omega B$, and so $B$ is $\Omega$-trivial.

COROLlary 1. Suppose that $A \stackrel{f}{\rightarrow} B \stackrel{g}{\rightarrow} C$ is an exact sequence of $\Omega$-normal $\mathfrak{\mathfrak { H }}$-objects. If $A$ and $C$ are both $\Omega$-trivial, then so is $B$.

Proof. From the sequence $A \stackrel{f}{\rightarrow} B \stackrel{g}{\rightarrow} C$ of $\Omega$-normal $\mathfrak{A}$-objects, we obtain the exact sequence

$$
0 \rightarrow \operatorname{Ker} g \rightarrow B \rightarrow \operatorname{Coker} f \rightarrow 0
$$

of $\Omega$-normal $\mathfrak{Q}$-objects, where, in addition, $\operatorname{Ker} g$ and Coker $f$ are $\Omega$-trivial. By the Lemma, $B$ is also $\Omega$-trivial.

COROLLARY 2. If $B$ is $\Omega$-trivial, then so are all its subobjects and all its factor objects.

COROLlary 3. The biproduct $A_{1} * A_{2} * \ldots * A_{n}$ is $\Omega$-trivial if and only if each $A_{i}$ is $\Omega$-trivial.

Proposition 2. Let $A$ be $\Omega$-normal and let $B$ be a subobject of $A$. Then $B$ is $\Omega$-trivial if and only if $B \leqq \Omega^{p} A$ for all $p \geqq 1$.

Proof. If $B$ is $\Omega$-trivial, then $B=\Omega B$, and so $B=\Omega^{p} B \leqq \Omega^{p} A$, for all $p \geqq 1$. Conversely, since $A$ is $\Omega$-normal, there exists a positive integer $n$ such that $\Omega^{n} A \cap B \leqq \Omega B$. Since $B \leqq \Omega^{n} A$, it follows that $B \leqq \Omega B$. That $B$ is $\Omega$-trivial now follows from Lemma 2 .

2. Regular sequences. Let $A$ belong to $\mathfrak{A}$ and let $B$ be a subobject of $A$. Further let $\gamma \in \mathscr{F}$. We use $B:{ }_{A} \gamma$ to denote the inverse image of $B$ with respect to $\gamma^{A}: A \rightarrow A$, so that 
$B:{ }_{A} \gamma$ is, in fact, the largest subobject $C$ of $A$ such that $\gamma C \leqq B$. It should be noted that if $D$ is a subobject of $B$, then

$$
\left(B:{ }_{A} \gamma\right) / D=B / D:_{A / D} \gamma
$$

and therefore, in particular

$$
\left(B:_{A} \gamma\right) / B=0:_{A / B} \gamma
$$

We also make frequent use of the trivial isomorphism

$$
A /(B+\gamma A) \approx(A \mid B) / \gamma(A / B) \text {. }
$$

For the next definition we use $\Gamma$ to denote the sequence $\gamma_{1}, \gamma_{2}, \ldots, \gamma_{t}$ of members of $\mathscr{F}$. $\Omega$ will denote a second finite sequence (not necessarily of the same length) whose members we do not need to specify. For $0 \leqq i \leqq t$ we use $\Gamma_{i}$ to denote the partial sequence $\gamma_{1}, \gamma_{2}, \ldots, \gamma_{i}$, and by $\Gamma_{0} A$ we shall understand the null subobject of $A$.

Definition. The sequence $\Gamma$ consisting of members $\gamma_{1}, \gamma_{2}, \ldots, \gamma_{t}$ of $\mathscr{F}$ will be said to be " $\Omega$-regular on $A$ " if $\left(\Gamma_{i-1} A:_{A} \gamma_{i}\right) / \Gamma_{i-1} A$ is $\Omega$-trivial for $1 \leqq i \leqq t$.

We shall use the convention that an empty sequence of members of $\mathscr{F}$ will always be $\Omega$-regular on $A$, whatever $A$ and $\Omega$ happen to be. We note that if $\gamma_{1}, \gamma_{2}, \ldots, \gamma_{t}$ is an $\Omega$-regular sequence on $A$, then it is also an $\Omega^{k}$-regular sequence on $A$, for every positive integer $k$.

The proof of the next lemma is entirely straightforward and will be omitted.

Lemma 5. Suppose that $0 \leqq m \leqq t$. Then in order that $\gamma_{1}, \gamma_{2}, \ldots, \gamma_{t}$ be $\Omega$-regular on $A$ it is necessary and sufficient that (i) $\gamma_{1}, \gamma_{2}, \ldots, \gamma_{m}$ be $\Omega$-regular on $A$ and (ii) $\gamma_{m+1}, \gamma_{m+2}, \ldots, \gamma_{t}$ be ת-regular on $A /\left(\gamma_{1} A+\gamma_{2} A+\ldots+\gamma_{m} A\right)$.

DEFINITION. We shall say that the sequence $\omega_{1}, \omega_{2}, \ldots, \omega_{s}$ of members of $\mathscr{F}$ is a " regular sequence on $A$ " if it is $\Omega$-regular on $A$, where $\Omega=\left\{\omega_{1}, \omega_{2}, \ldots, \omega_{s}\right\}$.

Suppose that $\omega_{1}, \omega_{2}, \ldots, \omega_{s}$ is a regular sequence on $A$ and that $\left\{v_{1}, v_{2}, \ldots, v_{s}\right\}$ is a permutation of $\{1,2, \ldots, s\}$. It will be shown (see Theorem 3 ) that, provided $A$ is $\Omega$-normal, then $\omega_{v_{1}}, \omega_{v_{2}}, \ldots, \omega_{v_{s}}$ is also a regular sequence on $A$. For the present we note that if $1 \leqq p<s$ it does not necessarily follow that $\omega_{1}, \omega_{2}, \ldots, \omega_{p}$ is a regular sequence on $A$. However we do have

Lemma 6. Let $\omega_{1}, \omega_{2}, \ldots, \omega_{s}$ be a regular sequence on the $\mathfrak{A}$-object $A$. Then, for each $i(0 \leqq i \leqq s), \omega_{i+1}, \omega_{i+2}, \ldots, \omega_{s}$ is a regular sequence on $A /\left(\omega_{1} A+\omega_{2} A+\ldots+\omega_{i} A\right)$.

Proof. We shall use $\Omega^{\prime}$ to denote the sequence $\omega_{i+1}, \omega_{i+2}, \ldots, \omega_{s}$. Furthermore, we shall let

$$
B=A /\left(\omega_{1} A+\omega_{2} A+\ldots+\omega_{i} A\right) .
$$

Let $L$ be a factor object of a subobject of $B$. Since $\omega_{1} B+\omega_{2} B+\ldots+\omega_{i} B=0$ we have $\omega_{1} L+\omega_{2} L+\ldots+\omega_{i} L=0$ and therefore $\Omega L=\Omega^{\prime} L$. We also have $\Omega L_{1}=\Omega^{\prime} L_{1}$ if $L_{1}$ is a subobject of $L$. Now from $\Omega L=\Omega^{\prime} L$ follows $\Omega^{m} L=\left(\Omega^{\prime}\right)^{m} L$ for all $m>0$. Hence if $L$ is $\Omega$-normal then it is $\Omega^{\prime}$-normal as well and if $L$ is $\Omega$-trivial it must also be $\Omega^{\prime}$-trivial. 
By Lemma $5, \omega_{i+1}, \omega_{i+2}, \ldots, \omega_{s}$ is $\Omega$-regular on $B$; that is to say, the objects

$$
\frac{\omega_{i+1} B+\ldots+\omega_{p-1} B:_{B} \omega_{p}}{\omega_{i+1} B+\ldots+\omega_{p-1} B} \quad(i+1 \leqq p \leqq s)
$$

are $\Omega$-trivial. The above remarks now show they are all $\Omega^{\prime}$-trivial, which is what we have to prove.

LEMMA 7. Let $\omega_{1}, \ldots, \omega_{s-1}, \omega_{s}$ be a regular sequence on the $\Omega$-normal $\mathfrak{U}$-object $A$, and let $p>0$ be an integer. Then $\omega_{1}, \ldots, \omega_{s-1}, \omega_{s}^{p}$ is a regular sequence on $A$.

Proof. First of all we shall show that if $\gamma, \gamma^{\prime} \in \mathscr{F}$ are such that $0:{ }_{A} \gamma$ and $0:{ }_{A} \gamma^{\prime}$ are both $\Omega$-trivial then $0:_{A} \gamma \gamma^{\prime}$ is also $\Omega$-trivial. We consider the exact sequence of $\Omega$-normal $\mathcal{A}$-objects

$$
0 \rightarrow 0:{ }_{B} \gamma^{\prime} \rightarrow B \rightarrow \gamma^{\prime} B \rightarrow 0 \text {. }
$$

Here $B=0:{ }_{A} \gamma \gamma^{\prime}$. Now $0:{ }_{B} \gamma^{\prime}$ is a subobject of $0:{ }_{A} \gamma^{\prime}$, and so $0:_{B} \gamma^{\prime}$ is $\Omega$-trivial. Also

$$
\gamma\left(\gamma^{\prime} B\right)=\left(\gamma \gamma^{\prime}\right) B=0 .
$$

Thus $\gamma^{\prime} B$ is a subobject of $0:{ }_{A} \gamma$, and so $\gamma^{\prime} B$ is $\Omega$-trivial. That $B$ is $\Omega$-trivial follows from Lemma 4.

By induction using this result, we see that if $0:{ }_{A} \gamma$ is $\Omega$-trivial then $0:{ }_{A} \gamma^{p}$ is $\Omega$-trivial for all $p>0$.

Let $\Omega^{\prime}=\left\{\omega_{1}, \ldots, \omega_{s-1}, \omega_{s}^{p}\right\}$. For any object $X$ we have

$$
\Omega^{p} X \leqq \Omega^{\prime} X \leqq \Omega X
$$

and therefore

$$
\Omega^{m p} X \leqq \Omega^{\prime m} X \leqq \Omega^{m} X,
$$

for all $m>0$. It follows readily that if $X$ is $\Omega$-normal, resp. $\Omega$-trivial, then it is also $\Omega^{\prime}$-normal, resp. $\Omega^{\prime}$-trivial. Accordingly it is enough to show that

$$
\left(\left(\omega_{1} A+\ldots+\omega_{i-1} A\right):{ }_{A} \omega_{i}\right) /\left(\omega_{1} A+\ldots+\omega_{i-1} A\right)
$$

is $\Omega$-trivial for $i=1,2, \ldots, s-1$ (which we know already) and, setting

$$
B=\omega_{1} A+\omega_{2} A+\ldots+\omega_{s-1} A \text {, }
$$

that

$$
\left(\left(\omega_{1} A+\ldots+\omega_{s-1} A\right):{ }_{A} \omega_{s}^{p}\right) /\left(\omega_{1} A+\ldots+\omega_{s-1} A\right)=0:_{B} \omega_{s}^{p}
$$

is $\Omega$-trivial as well. However $0:_{B} \omega_{s}$ is $\Omega$-trivial by hypothesis and therefore the latter requirement follows by virtue of the remarks made above.

3. Regular sequences and the Koszul complex. Let $A$ be an object of $\mathfrak{A}$ and $\omega_{1}, \omega_{2}, \ldots, \omega_{s}$ members of $\mathscr{F}$. It is then possible to form the Koszul complex $K\left(\omega_{1}, \omega_{2}, \ldots, \omega_{s} \mid A\right)$ of $A$ with respect to $\omega_{1}, \omega_{2}, \ldots, \omega_{s}$. We recall that the $\mu$ th component $K_{\mu}\left(\omega_{1}, \omega_{2}, \ldots, \omega_{s} \mid A\right)$ of this complex is the biproduct of $\left(\begin{array}{l}s \\ \mu\end{array}\right)$ terms all equal to $A$. Here $\left(\begin{array}{l}s \\ \mu\end{array}\right)$ denotes the usual binomial coefficient. Thus $K_{\mu}\left(\omega_{1}, \omega_{2}, \ldots, \omega_{s} \mid A\right)=0$ if either $\mu<0$ or $\mu>s$. 
Suppose that $0 \leqq \mu \leqq s$ and let $i_{1}, i_{2}, \ldots, i_{\mu}$, here and in what follows, be a typical sequence of integers satisfying $1 \leqq i_{1}<i_{2}<\ldots<i_{\mu} \leqq s$. We use these sequences to label the terms in the biproduct $K_{\mu}\left(\omega_{1}, \omega_{2}, \ldots, \omega_{s} \mid A\right)$ so that $A_{i_{1} i_{2} \ldots i_{\mu}}$ denotes $A$ considered as the $\left(i_{1}, i_{2}, \ldots, i_{\mu}\right)$ th term. Accordingly there is an embedding morphism

$$
\sigma_{i_{1} i_{2} \ldots i_{\mu}}: A \rightarrow K_{\mu}\left(\omega_{1}, \omega_{2}, \ldots, \omega_{s} \mid A\right)
$$

and a projection morphism

$$
\pi_{i_{1} i_{2} \ldots i_{\mu}}: K_{\mu}\left(\omega_{1}, \omega_{2}, \ldots, \omega_{s} \mid A\right) \rightarrow A
$$

associated with $A_{i_{1} i_{2} \ldots i_{\mu}}$.

Suppose next that $1 \leqq \mu \leqq s$ and let $j_{1}, j_{2}, \ldots, j_{\mu-1}$ be integers satisfying

$$
1 \leqq j_{1}<j_{2}<\ldots<j_{\mu-1} \leqq s
$$

Then the boundary morphism

$$
d_{\mu}: K_{\mu}\left(\omega_{1}, \omega_{2}, \ldots, \omega_{s} \mid A\right) \rightarrow K_{\mu-1}\left(\omega_{1}, \omega_{2}, \ldots, \omega_{s} \mid A\right)
$$

is characterized by the fact that

$$
\pi_{j_{1} j_{2} \ldots j_{\mu-1}} \cdot d_{\mu} \cdot \sigma_{i_{1} i_{2} \ldots i_{\mu}}: A \rightarrow A
$$

is null if $\left\{j_{1}, j_{2}, \ldots, j_{\mu-1}\right\}$ is not contained in $\left\{i_{1}, i_{2}, \ldots, i_{\mu}\right\}$ and is $\left(-1^{A}\right)^{p-1} \omega_{i_{p}}^{A}$ if $\left\{j_{1}, j_{2}, \ldots, j_{\mu-1}\right\}$ is the result of deleting $i_{p}$ from $\left\{i_{1}, i_{2}, \ldots, i_{\mu}\right\}$.

We shall need a number of properties of the Koszul complex. Proofs of these will be found in [3, Chapter 8] for the case of modules and it is a straightforward matter to adapt these results so that they apply to a general abelian category.

Let $\left\{v_{1}, v_{2}, \ldots, v_{s}\right\}$ be a permutation of $\{1,2, \ldots, s\}$. Then [3, Proposition 1, p. 361] the complexes $K\left(\omega_{v_{1}}, \omega_{v_{2}}, \ldots, \omega_{v_{s}} \mid A\right)$ and $K\left(\omega_{1}, \omega_{2}, \ldots, \omega_{s} \mid A\right)$ are isomorphic and therefore, for each $\mu$, we have an isomorphism

$$
H_{\mu} K\left(\omega_{v_{1}}, \omega_{v_{2}}, \ldots, \omega_{v_{s}} \mid A\right) \approx H_{\mu} K\left(\omega_{1}, \omega_{2}, \ldots, \omega_{s} \mid A\right)
$$

between their $\mu$ th homology modules. A less familiar result says that $K\left(\omega_{1}, \omega_{2}, \ldots, \omega_{s} \mid A\right)$ remains unchanged to within isomorphism if we change the signs of an arbitrary number of the $\omega_{i}$. In view of the above remarks this will be clear if it is shown that $K\left(\omega_{1}, \ldots, \omega_{s-1}, \omega_{s} \mid A\right)$ and $K\left(\omega_{1}, \ldots, \omega_{s-1},-\omega_{s} \mid A\right)$ are isomorphic. An isomorphism $\phi$ may, in fact, be obtained by taking

$$
\phi_{\mu}: K_{\mu}\left(\omega_{1}, \ldots, \omega_{s-1}, \omega_{s} \mid A\right) \rightarrow K_{\mu}\left(\omega_{1}, \ldots, \omega_{s-1},-\omega_{s} \mid A\right)
$$

to be the biproduct of the morphisms

$$
\phi_{i_{1} i_{2} \ldots i_{\mu}}: A_{i_{1} i_{2} \ldots i_{\mu}} \rightarrow A_{i_{1} i_{2} \ldots i_{\mu}},
$$

where $\phi_{i_{1} i_{2} \ldots i_{\mu}}$ is $1^{A}$ if $i_{\mu} \neq s$ and $-1^{A}$ if $i_{\mu}=s$. It follows, in particular, that the complexes $\boldsymbol{K}\left(\omega_{1}, \omega_{2}, \omega_{3}, \ldots, \omega_{s} \mid A\right)$ and $K\left(\omega_{1},-\omega_{2}, \omega_{3}, \ldots,(-1)^{s-1} \omega_{s} \mid A\right)$ are isomorphic and therefore, for each $\mu$, we have an isomorphism

$$
H_{\mu} K\left(\omega_{1}, \omega_{2}, \omega_{3}, \ldots, \omega_{s} \mid A\right) \approx H_{\mu} K\left(\omega_{1},-\omega_{2}, \omega_{3}, \ldots,(-1)^{s-1} \omega_{s} \mid A\right) .
$$


An important property of the Koszul complex [3, Theorem 3, p. 364] states that, for $1 \leqq i \leqq s$ and arbitrary $\mu$,

$$
\omega_{i} H_{\mu} K\left(\omega_{1}, \omega_{2}, \ldots, \omega_{s} \mid A\right)=0 .
$$

Hence if the identity element of $\mathscr{F}$ occurs among $\omega_{1}, \omega_{2}, \ldots, \omega_{s}$, then

$$
H_{\mu} K\left(\omega_{1}, \omega_{2}, \ldots, \omega_{s} \mid A\right)=0
$$

for all $\mu$.

LEMmA 8. Suppose that $0:_{A} \omega_{s}$ is $\Omega$-trivial. Then, for each $\mu, H_{\mu} K\left(\omega_{1}, \ldots, \omega_{s-1}, \omega_{s} \mid A\right)$ and $H_{\mu} K\left(\omega_{1}, \ldots, \omega_{s-1} \mid A / \omega_{s} A\right)$ are isomorphic.

Proof. $X$ will denote the complex whose $\mu$ th component object is

$$
X_{\mu}=K_{\mu-1}\left(\omega_{1}, \ldots, \omega_{s-1} \mid A / \omega_{s} A\right)
$$

and whose boundary morphism $X_{\mu} \rightarrow X_{\mu-1}$ is the boundary morphism

$$
K_{\mu-1}\left(\omega_{1}, \ldots, \omega_{s-1} \mid A / \omega_{s} A\right) \rightarrow K_{\mu-2}\left(\omega_{1}, \ldots, \omega_{s-1} \mid A / \omega_{s} A\right)
$$

of the complex $K\left(\omega_{1}, \ldots, \omega_{s-1} \mid A / \omega_{s} A\right)$. Then

$$
H_{\mu}(X)=H_{\mu-1} K\left(\omega_{1}, \ldots, \omega_{s-1} \mid A / \omega_{s} A\right),
$$

where $H_{\mu}(X)$ is the $\mu$ th homology object of the complex $X$.

There is an exact sequence of complexes

$$
K\left(\omega_{1}, \ldots, \omega_{s-1}, \omega_{s} \mid A\right) \stackrel{f}{\rightarrow} K\left(\omega_{1}, \ldots, \omega_{s-1}, 1 \mid A\right) \stackrel{g}{\rightarrow} X \rightarrow 0 .
$$

The details of the constructions of the morphisms $f$ and $g$ are to be found in [3, pp. 366-8]. These details are not all necessary for our present purposes. However we should note that both $K_{\mu}\left(\omega_{1}, \ldots, \omega_{s-1}, \omega_{s} \mid A\right)$ and $K_{\mu}\left(\omega_{1}, \ldots, \omega_{s-1}, 1 \mid A\right)$ are biproducts of $\left(_{\mu}^{s}\right)$ copies of $A$, and that $f_{\mu}$ involves applying $1^{A}$ to some of these copies of $A$, while applying $\omega_{s}^{A}$ to the remainder. Hence $\operatorname{Ker} f_{\mu}$ is a biproduct of a finite number of copies of $0:_{A} \omega_{s}$ and is therefore $\Omega$-trivial (Lemma 4, Corollary 3).

From (3.5) we obtain the exact sequences of complexes

and

$$
0 \rightarrow \operatorname{Ker} f \rightarrow K\left(\omega_{1}, \ldots, \omega_{s-1}, \omega_{s} \mid A\right) \rightarrow \operatorname{Im} f \rightarrow 0
$$

$$
0 \rightarrow \operatorname{Im} f \rightarrow K\left(\omega_{1}, \ldots, \omega_{s-1}, 1 \mid A\right) \rightarrow X \rightarrow 0 .
$$

By the remark immediately preceding the Lemma, all the homology objects of

$$
K\left(\omega_{1}, \ldots, \omega_{s-1}, 1 \mid A\right)
$$

are null. Thus the homology sequence of (3.7) reduces to a series of isomorphisms,

$$
H_{\mu+1}(X) \approx H_{\mu}(\operatorname{Im} f) \text {, }
$$

which, by (3.4), may be rewritten as

$$
H_{\mu} K\left(\omega_{1}, \ldots, \omega_{s-1} \mid A / \omega_{s} A\right) \approx H_{\mu}(\operatorname{Im} f)
$$


The homology sequence of (3.6) yields an exact sequence

$$
H_{\mu}(\operatorname{Ker} f) \rightarrow H_{\mu} K\left(\omega_{1}, \ldots, \omega_{s-1}, \omega_{s} \mid A\right) \stackrel{\phi_{\mu}}{\rightarrow} H_{\mu}(\operatorname{Im} f) \rightarrow H_{\mu-1}(\operatorname{Ker} f) .
$$

It remains to show that each $\phi_{\mu}$ is an isomorphism.

Firstly we note that $H_{\mu}(\operatorname{Ker} f)$ and $H_{\mu-1}(\operatorname{Ker} f)$ are both $\Omega$-trivial, being factor objects of subobjects of $\operatorname{Ker} f_{\mu}$ and $\operatorname{Ker} f_{\mu-1}$ respectively. Thus $\operatorname{Ker} \phi_{\mu}$ and $\operatorname{Coker} \phi_{\mu}$ are both $\Omega$-trivial. Since $\operatorname{Ker} \phi_{\mu}$ is a subobject of $H_{\mu} K\left(\omega_{1}, \ldots, \omega_{s-1}, \omega_{s} \mid A\right)$, it follows from (3.3) that

$$
\operatorname{Ker} \phi_{\mu}=\Omega \cdot \operatorname{Ker} \phi_{\mu} \leqq \Omega \cdot H_{\mu} K\left(\omega_{1}, \ldots, \omega_{s-1}, \omega_{s} \mid A\right)=0
$$

Again, $\omega_{1}, \omega_{2}, \ldots, \omega_{s-1}$ all annihilate $H_{\mu} K\left(\omega_{1}, \ldots, \omega_{s-1} \mid A / \omega_{s} A\right)$, and

$$
\omega_{s} \cdot H_{\mu} K\left(\omega_{1}, \ldots, \omega_{s-1} \mid A / \omega_{s} A\right)=0
$$

since $\omega_{s}$ annihilates $A / \omega_{s} A$. By (3.8) it follows that

$$
\Omega \cdot H_{\mu}(\operatorname{Im} f) \approx \Omega \cdot H_{\mu} K\left(\omega_{1}, \ldots, \omega_{s-1} \mid A / \omega_{s} A\right)=0 .
$$

From this we may deduce that $\Omega \cdot \operatorname{Coker} \phi_{\mu}=0$, so that Coker $\phi_{\mu}=0$, since Coker $\phi_{\mu}$ is $\Omega$-trivial. Thus $\phi_{\mu}$ is an isomorphism, and so

$$
H_{\mu} K\left(\omega_{1}, \ldots, \omega_{s-1}, \omega_{s} \mid A\right) \approx H_{\mu} K\left(\omega_{1}, \ldots, \omega_{s-1} \mid A / \omega_{s} A\right) .
$$

This is the required result.

Proposition 3. Let $A$ be an $\mathfrak{A}$-object, and let $\omega_{1}, \omega_{2}, \ldots, \omega_{s}$ be a regular sequence on $A$. Then $H_{\mu} K\left(\omega_{1}, \omega_{2}, \ldots, \omega_{s} \mid A\right)=0$ for all $\mu \neq 0$.

Proof. We shall use induction on $s$. If $s=0$ the result is obviously true. We therefore assume that $s \geqq 1$ and make the obvious inductive assumption. Now $0:{ }_{A} \omega_{1}$ is $\Omega$-trivial. By (3.1) and Lemma 8, there are isomorphisms

$$
H_{\mu} K\left(\omega_{1}, \omega_{2}, \ldots, \omega_{s} \mid A\right) \approx H_{\mu} K\left(\omega_{2}, \ldots, \omega_{s}, \omega_{1} \mid A\right) \approx H_{\mu} K\left(\omega_{2}, \ldots, \omega_{s} \mid A / \omega_{1} A\right)
$$

for all $\mu$. However, by Lemma $6, \omega_{2}, \ldots, \omega_{s}$ is a regular sequence on $A / \omega_{1} A$. Thus $H_{\mu} K\left(\omega_{2}, \ldots, \omega_{s} \mid A / \omega_{1} A\right)=0$ for all $\mu \neq 0$, from which the proposition follows.

LEMMA 9. Let $A$ be an $\Omega$-normal $\mathfrak{A}$-object and let $1 \leqq m \leqq s$ be an integer. If $H_{\mu} K\left(\omega_{1}, \ldots, \omega_{m-1}, \omega_{m} \mid A\right)$ is $\Omega$-trivial for some $\mu$ then $H_{\mu} K\left(\omega_{1}, \ldots, \omega_{m-1} \mid A\right)$ is also $\Omega$-trivial.

Proof. It is a standard property $\dagger$ of the Koszul complex that the homology objects of the Koszul complexes $K\left(\omega_{1}, \ldots, \omega_{m-1} \mid A\right)$ and $K\left(\omega_{1}, \ldots, \omega_{m-1}, \omega_{m} \mid A\right)$ are connected through an exact sequence

$$
H_{\mu} K\left(\omega_{1}, \ldots, \omega_{m-1} \mid A\right) \stackrel{\Delta_{\mu}}{\rightarrow} H_{\mu} K\left(\omega_{1}, \ldots, \omega_{m-1} \mid A\right) \stackrel{\xi_{\mu}}{\rightarrow} H_{\mu} K\left(\omega_{1}, \ldots, \omega_{m-1}, \omega_{m} \mid A\right) .
$$

Here $\Delta_{\mu}$ is the morphism induced by $(-1)^{\mu} \omega_{m}$. By Lemmas 2 and 3, each of the 2f-objects in 
the above sequence is $\Omega$-normal. Now $\operatorname{Coker} \Delta_{\mu}=\operatorname{Coim} \xi_{\mu}=\operatorname{Im} \xi_{\mu}$, which is a subobject of $H_{\mu} K\left(\omega_{1}, \ldots, \omega_{m-1}, \omega_{m} \mid A\right)$ and hence is $\Omega$-trivial. Thus

$$
\operatorname{Coker} \Delta_{\mu}=\Omega \cdot \operatorname{Coker} \Delta_{\mu}
$$

Let $g$ be the natural epimorphism

$$
g: H_{\mu} K\left(\omega_{1}, \ldots, \omega_{m-1} \mid A\right) \rightarrow \text { Coker } \Delta_{\mu} .
$$

Then $g\left(H_{\mu} K\left(\omega_{1}, \ldots, \omega_{m-1} \mid A\right)\right)=\Omega \cdot\left(g\left(H_{\mu} K\left(\omega_{1}, \ldots, \omega_{m-1} \mid A\right)\right)\right)$, so that

$$
H_{\mu} K\left(\omega_{1}, \ldots, \omega_{m-1} \mid A\right)+\operatorname{Ker} g=\Omega \cdot H_{\mu} K\left(\omega_{1}, \ldots, \omega_{m-1} \mid A\right)+\operatorname{Ker} g .
$$

However $\operatorname{Ker} g=\operatorname{Im} \Delta_{\mu}=\omega_{m} \cdot H_{\mu} K\left(\omega_{1}, \ldots, \omega_{m-1} \mid A\right)$, so that

$$
H_{\mu} K\left(\omega_{1}, \ldots, \omega_{m-1} \mid A\right)=\Omega \cdot H_{\mu} K\left(\omega_{1}, \ldots, \omega_{m-1} \mid A\right),
$$

and hence $H_{\mu} K\left(\omega_{1}, \ldots, \omega_{m-1} \mid A\right)$ is $\Omega$-trivial as required.

LEMMA 10. Let $A$ be an $\Omega$-normal $\mathfrak{A}$-object and let $1 \leqq t \leqq s$ be an integer. If $H_{p} K\left(\omega_{1}, \omega_{2}, \ldots, \omega_{t} \mid A\right)$ is $\Omega$-trivial for some $p \geqq 0$ then $H_{q} K\left(\omega_{1}, \omega_{2}, \ldots, \omega_{t} \mid A\right)$ is also $\Omega$-trivial whenever $q \geqq p$.

Proof. The proof will be by induction on $t$.

(i) The case $t=1$. If $p \geqq 1$ the result is obvious. We shall therefore assume $p=0$. Now $H_{0} K\left(\omega_{1} \mid A\right)=A / \omega_{1} A$. Hence, by assumption, $\left(A / \omega_{1} A\right)=\Omega\left(A / \omega_{1} A\right)$. Thus $A=\Omega A$, so that $A$ is $\Omega$-trivial. $0:{ }_{A} \omega_{1}$, being a subobject of $A$, is also $\Omega$-trivial, so that $H_{1}\left(\omega_{1} \mid A\right)$ is $\Omega$-trivial. The result follows for the case $t=1$.

(ii) The case $t>1$. We shall make the obvious assumption concerning the inductive variable $t$. By Lemma $9, H_{p} K\left(\omega_{1}, \omega_{2}, \ldots, \omega_{t-1} \mid A\right)$ is $\Omega$-trivial, so that

$$
H_{q} K\left(\omega_{1}, \omega_{2}, \ldots, \omega_{t-1} \mid A\right)
$$

is $\Omega$-trivial for all $q \geqq p$. Now it is well-known [3, Proposition 2, p. 365] that there exists an exact sequence

$H_{q} K\left(\omega_{1}, \ldots, \omega_{t-1} \mid A\right) \rightarrow H_{q} K\left(\omega_{1}, \ldots, \omega_{t-1}, \omega_{t} \mid A\right) \rightarrow H_{q-1} K\left(\omega_{1}, \ldots, \omega_{t-1} \mid A\right)$.

From this sequence and from Lemma 4, Corollary 1 , it follows that $H_{q} K\left(\omega_{1}, \ldots, \omega_{t-1}, \omega_{t} \mid A\right)$ is $\Omega$-trivial for all $q>p$. The result of the Lemma is thus established.

TheOREM 1. Let $A$ be an $\Omega$-normal $\mathfrak{A}$-object, where $\Omega=\left\{\omega_{1}, \omega_{2}, \ldots, \omega_{s}\right\}$. If, for some integer $p \geqq 0, H_{p} K\left(\omega_{1}, \omega_{2}, \ldots, \omega_{s} \mid A\right)=0$ then $H_{q} K\left(\omega_{1}, \omega_{2}, \ldots, \omega_{s} \mid A\right)=0$ for all $q \geqq p$.

Proof. By Lemma $10, H_{q} K\left(\omega_{1}, \omega_{2}, \ldots, \omega_{s} \mid A\right)$ is $\Omega$-trivial for all $q \geqq p$. Hence

$$
H_{q} K\left(\omega_{1}, \omega_{2}, \ldots, \omega_{s} \mid A\right)=\Omega \cdot H_{q} K\left(\omega_{1}, \omega_{2}, \ldots, \omega_{s} \mid A\right) .
$$

However, by (3.3), $\Omega \cdot H_{q} K\left(\omega_{1}, \omega_{2}, \ldots, \omega_{s} \mid A\right)=0$. Thus $H_{q} K\left(\omega_{1}, \omega_{2}, \ldots, \omega_{s} \mid A\right)=0$ for all $q \geqq p$. 
COROLlARY. $\dagger$ Let $R$ be a ring with identity element, $A$ a Noetherian $R$-module, and $\omega_{1}, \omega_{2}, \ldots, \omega_{s}$ central elements of $R$. If for some integer $p \geqq 0, H_{p} K\left(\omega_{1}, \omega_{2}, \ldots, \omega_{s} \mid A\right)=0$ then $H_{q} K\left(\omega_{1}, \omega_{2}, \ldots, \omega_{s} \mid A\right)=0$ for all $q \geqq p$.

The corollary follows immediately from Proposition 1 and the above Theorem.

THEOREM 2. Let the $\mathfrak{I}$-object $A$ be $\Omega$-normal, where $\Omega=\left\{\omega_{1}, \omega_{2}, \ldots, \omega_{s}\right\}$. Then the following statements are equivalent:

(a) $\omega_{1}, \omega_{2}, \ldots, \omega_{s}$ is a regular sequence on $A$,

(b) $H_{1} K\left(\omega_{1}, \omega_{2}, \ldots, \omega_{s} \mid A\right)=0$.

Proof. Proposition 3 shows that (a) implies (b) even when $A$ is not $\Omega$-normal. We shall show that, when $A$ is $\Omega$-normal, (b) implies (a) by using induction on $s$. We may suppose that $s \geqq 1$.

(i) The case $s=1$. Since $H_{1} K\left(\omega_{1} \mid A\right)=0:{ }_{A} \omega_{1}, H_{1} K\left(\omega_{1} \mid A\right)=0$ implies that $0:{ }_{A} \omega_{1}=0$, so that $\omega_{1}$ is a regular sequence on $A$.

(ii) The case $s>1$. We assume that the required result has been established for any sequence containing less than $s$ members.

Since $H_{1} K\left(\omega_{1}, \omega_{2}, \ldots, \omega_{s} \mid A\right)=0$, Lemma 9 shows that $H_{1} K\left(\omega_{1}, \omega_{2}, \ldots, \omega_{m} \mid A\right)$ is $\Omega$-trivial for $1 \leqq m \leqq s$, and in particular that $H_{1} K\left(\omega_{1} \mid A\right)$ is $\Omega$-trivial. Therefore $0:{ }_{A} \omega_{1}$ is $\Omega$-trivial, so that $\omega_{1}$ is an $\Omega$-regular sequence on $A$. By Lemma 8 there are isomorphisms

$$
H_{1} K\left(\omega_{1}, \omega_{2}, \ldots, \omega_{s} \mid A\right) \approx H_{1} K\left(\omega_{2}, \ldots, \omega_{s}, \omega_{1} \mid A\right) \approx H_{1} K\left(\omega_{2}, \ldots, \omega_{s} \mid A / \omega_{1} A\right)
$$

and so $H_{1} K\left(\omega_{2}, \ldots, \omega_{s} \mid A / \omega_{1} A\right)=0$. Now $A / \omega_{1} A$ is $\Omega$-normal, and $\omega_{1}\left(A / \omega_{1} A\right)=0$, from which it follows that $A / \omega_{1} A$ is $\Omega^{\prime}$-normal, where $\Omega^{\prime}=\left\{\omega_{2}, \omega_{3}, \ldots, \omega_{s}\right\}$. Thus, by inductive hypothesis, $\omega_{2}, \omega_{3}, \ldots, \omega_{s}$ is a regular sequence on $\left(A / \omega_{1} A\right)=B$. Hence each of

$$
\left(\Omega_{i-1}^{\prime} B:_{B} \omega_{i}\right) / \Omega_{i-1}^{\prime} B \quad(2 \leqq i \leqq s)
$$

is $\Omega^{\prime}$-trivial, where $\Omega_{i-1}^{\prime}=\left\{\omega_{2}, \omega_{3}, \ldots, \omega_{i-1}\right\}$ for $3 \leqq i \leqq s$, and $\Omega_{1}^{\prime} B=0$. In particular, for $2 \leqq i \leqq s$,

$$
\left\{\left(\Omega_{i-1}^{\prime} B:_{B} \omega_{i}\right) / \Omega_{i-1}^{\prime} B\right\}=\Omega^{\prime}\left\{\left(\Omega_{i-1}^{\prime} B:_{B} \omega_{i}\right) / \Omega_{i-1}^{\prime} B\right\},
$$

from which it follows that

$$
\left.\left\{\left(\Omega_{i-1}^{\prime} B:_{B} \omega_{i}\right) / \Omega_{i-1}^{\prime} B\right\}=\Omega_{\{}^{\prime}\left(\Omega_{i-1}^{\prime} B:_{B} \omega_{i}\right) / \Omega_{i-1}^{\prime} B\right\} .
$$

Since each of

$$
\left(\Omega_{i-1}^{\prime} B:{ }_{B} \omega_{i}\right) / \Omega_{i-1}^{\prime} B \quad(2 \leqq i \leqq s)
$$

is a factor object of a subobject of a factor object of $A$, these $\mathfrak{A}$-objects are all $\Omega$-normal. It follows therefore that

$$
\left(\Omega_{i-1}^{\prime} B:_{B} \omega_{i}\right) / \Omega_{i-1}^{\prime} B
$$

$\dagger \mathrm{cf}$. [1, Proposition 2.6, p. 632]. 
is $\Omega$-trivial for $2 \leqq i \leqq s$, so that $\left\{\omega_{2}, \omega_{3}, \ldots, \omega_{s}\right\}$ is an $\Omega$-regular sequence on $A / \omega_{1} A$. By Lemma $5, \omega_{1}, \omega_{2}, \ldots, \omega_{s}$ is an $\Omega$-regular sequence on $A$, which is the desired result.

THeOREM 3. Suppose that $\Omega=\left\{\omega_{1}, \omega_{2}, \ldots, \omega_{s}\right\}$, and that $\left\{v_{1}, v_{2}, \ldots, v_{s}\right\}$ is a permutation of $\{1,2, \ldots, s\}$. If $\omega_{1}, \omega_{2}, \ldots, \omega_{s}$ is a regular sequence on the $\Omega$-normal $\mathfrak{A}$-object $A$ then $\omega_{v_{1}}, \omega_{v_{2}}, \ldots, \omega_{v_{s}}$ is also a regular sequence on $A$.

Proof. The desired result follows at once from Theorem 2 and the isomorphism (3.1)

$$
H_{1} K\left(\omega_{1}, \omega_{2}, \ldots, \omega_{s} \mid A\right) \approx H_{1} K\left(\omega_{v_{1}}, \omega_{v_{2}}, \ldots, \omega_{v_{s}} \mid A\right) .
$$

THEOREM 4. Suppose that $\Omega=\left\{\omega_{1}, \omega_{2}, \ldots, \omega_{s}\right\}$, and that $p_{1}, p_{2}, \ldots, p_{s}$ are positive integers. If $\omega_{1}, \omega_{2}, \ldots, \omega_{s}$ is a regular sequence on the $\Omega$-normal $\mathfrak{A}$-object $A$, then $\omega_{1}^{p_{1}}, \omega_{2}^{p_{2}}, \ldots, \omega_{s}^{p_{s}}$ is also a regular sequence on $A$.

Proof. By Lemma $7, \omega_{1}, \omega_{2}, \ldots, \omega_{s}^{p_{s}}$ is a regular sequence on $A$. Also, from the proof of Lemma $7, A$ is $\left\{\omega_{1}, \ldots, \omega_{s-1}, \omega_{s}^{p_{s}}\right\}$-normal. Thus we may permute $\left\{\omega_{1}, \ldots, \omega_{s-1}, \omega_{s}^{p_{s}}\right\}$, by Theorem 3 , and the sequence so obtained will still be regular on $A$. Repeated applications of this observation now yield the required result.

4. The dual theory. Let $G: \mathfrak{I} \rightarrow \mathfrak{I}^{*}$ be a duality between abelian categories. Let $\mathscr{F}$ and $\mathscr{F F}^{*}$ denote respectively the classes of natural transformations of the identity functors on $\mathfrak{A}$ and $\mathfrak{I}^{*}$. We shall use $A^{*}$ and $f^{*}$ to denote the $\mathfrak{I}^{*}$-object and $\mathfrak{I}^{*}$-morphism which correspond under the duality $G$ to $A$ and $f$ respectively. $C$ then

It may be easily verified that if $A_{1}, A_{2}, \ldots, A_{n}$ is a sequence of subobjects of the $\mathfrak{A}$-object

$$
\left(\bigcap_{i=1}^{n} A_{i}\right)^{*}=C^{*} / \sum_{i=1}^{n}\left(C / A_{i}\right)^{*}
$$

and

$$
\left(\sum_{i=1}^{n} A_{i}\right)^{*}=C^{*} / \bigcap_{i=1}^{n}\left(C / A_{i}\right)^{*}
$$

There is a one-to-one correspondence between the members of $\mathscr{F}$ and the members of $\mathscr{F F}^{*}$. This correspondence is such that if $\omega \in \mathscr{F}$ and $\omega^{*} \in \mathscr{F}^{*}$ correspond then

$$
\left(\omega^{*}\right)^{A *}=\left(\omega^{A}\right)^{*} \text {, }
$$

for all $\mathfrak{A}$-objects $A$. Under the above correspondence it may be readily seen that $\gamma \delta$ and $\gamma^{*} \delta^{*}$ correspond.

If $\Omega$ denotes the sequence $\left\{\omega_{1}, \omega_{2}, \ldots, \omega_{s}\right\}$ of members of $\mathscr{F}$ then we shall use $\Omega^{*}$ to denote the sequence $\left\{\omega_{1}^{*}, \omega_{2}^{*}, \ldots, \omega_{s}^{*}\right\}$ of members of $\mathscr{F}^{*}$. Here it is assumed that $\omega_{i}$ and $\omega_{i}^{*}$ correspond. It should be noted that $\left(\Omega^{*}\right)^{r}=\left(\Omega^{r}\right)^{*}$ for every positive integer $r$.

Given an $\mathcal{A}$-object $A$, a subobject $B$ of $A$, and a sequence $\Omega=\left\{\omega_{1}, \omega_{2}, \ldots, \omega_{s}\right\}$ of members of $\mathscr{F}$, we put

$$
B:{ }_{A} \Omega=\bigcap_{i=1}^{s}\left(B:{ }_{A} \omega_{i}\right)
$$


LEMMA 11. In the above situation

and

$$
\left(B:{ }_{A} \Omega\right)^{*}=A^{*} / \Omega^{*}(A / B)^{*}
$$

$$
\Omega^{*}(A / B)^{*}=\left\{A /\left(B:{ }_{A} \Omega\right)\right\}^{*} .
$$

Proof. Firstly we note that $B:{ }_{A} \omega_{i}$ is the kernel of the composite morphism

$$
A \stackrel{\omega_{i}^{A}}{\rightarrow} A \rightarrow A / B
$$

so that $\left(B:{ }_{A} \omega_{i}\right)^{*}$ is the cokernel of

$$
(A / B)^{*} \rightarrow A^{*} \rightarrow A^{*},
$$

where $A^{*} \rightarrow A^{*}$ is the morphism $\left(\omega_{i}^{*}\right)^{A^{*}}$. This shows that $\left(B:_{A} \omega_{i}\right)^{*}=A^{*} j \omega_{i}^{*}(A / B)^{*}$, and now from the exact sequence

$$
0 \rightarrow\left(A /\left(B:{ }_{A} \omega_{i}\right)\right)^{*} \rightarrow A^{*} \rightarrow\left(B:{ }_{A} \omega_{i}\right)^{*} \rightarrow 0
$$

we conclude that

$$
\left(A /\left(B:{ }_{A} \omega_{i}\right)\right)^{*}=\omega_{i}^{*}(A / B)^{*}
$$

It follows from (4.1) that

$$
\begin{aligned}
\left(B:_{A} \Omega\right)^{*} & =A^{*} / \sum_{i=1}^{s}\left(A /\left(B:_{A} \omega_{i}\right)\right)^{*} \\
& =A^{*} / \sum_{i=1}^{s} \omega_{i}^{*}(A / B)^{*}=A^{*} / \Omega^{*}(A / B)^{*}
\end{aligned}
$$

Hence

$$
\begin{aligned}
\Omega^{*}(A / B)^{*} & =\operatorname{Ker}\left\{A^{*} \rightarrow\left(B:_{A} \Omega\right)^{*}\right\} \\
& =\left(\operatorname{Coker}\left\{\left(B:{ }_{A} \Omega\right) \rightarrow A\right\}\right)^{*}=\left(A /\left(B:_{A} \Omega\right)\right)^{*} .
\end{aligned}
$$

The Lemma is thus proved.

After the above preliminaries we are now in a position to give the definitions of the concepts dual to $\Omega$-normality, $\Omega$-triviality, and regular sequences, and to interpret these new concepts in terms of objects of the original category $\mathfrak{U}$. We begin this by making the

Defintion. Let $\Omega=\left\{\omega_{1}, \omega_{2}, \ldots, \omega_{s}\right\}$ be a sequence of members of $\mathscr{F}$. The $\mathfrak{1}$-object $A$ is said to be " $\Omega$-conormal" if and only if the $\mathfrak{U}^{*}$-object $A^{*}$ is $\Omega^{*}$-normal.

Proposition 4. The $\mathfrak{A}$-object $A$ is $\Omega$-conormal when and only when it satisfies the following condition:

If $X$ is any subobject of $A$ then there exists a positive integer $p$, dependent upon $X$, such that

$$
\left(X:{ }_{A} \Omega\right) \leqq X+\left(0:{ }_{A} \Omega^{p}\right)
$$

as subobjects of $A$. 
Proof. Let $X$ be any subobject of $A$, and let $B=A / X$. The proposition follows at one from the equivalence of the following statements:

(i) $\left(\Omega^{*}\right)^{p} A^{*} \cap B^{*} \leqq \Omega^{*} B^{*}$ as subobjects of $A^{*}$,

(ii) $\left\{\left(\Omega^{*}\right)^{p} A^{*} \cap B^{*}\right\}^{*} \leqq\left\{\Omega^{*} B^{*}\right\}^{*}$ as factor objects of $A$,

(iii) $A /\left\{\left(A^{*} /\left(\Omega^{*}\right)^{p} A^{*}\right)^{*}+\left(A^{*} / B^{*}\right)^{*}\right\} \leqq A /\left(X:{ }_{A} \Omega\right)$ as factor objects of $A$ (by (4.1) and (4.5)),

(iv) $A /\left\{\left(0:{ }_{A} \Omega^{p}\right)+X\right\} \leqq A /\left(X:{ }_{A} \Omega\right)$ as factor objects of $A$ (by (4.4)),

(v) $\left(X:{ }_{A} \Omega\right) \leqq X+\left(0:{ }_{A} \Omega^{p}\right)$ as subobjects of $A$.

Definition. The $\mathfrak{Y}$-object $A$ is said to be " $\Omega$-cotrivial" if and only if the $\mathfrak{U l}^{*}$-object $A^{*}$ is $\Omega^{*}$-trivial.

Proposition 5. A necessary and sufficient condition for $A$ to be $\Omega$-cotrivial is that $A$ be $\Omega$-conormal and $0:{ }_{A} \Omega=0$.

Proof. This follows immediately from the fact (see (4.4)) that $A^{*}=\Omega^{*} A^{*}$ if and only if $0:{ }_{A} \Omega=0$.

Definition. The sequence $\omega_{1}, \omega_{2}, \ldots, \omega_{s}$ is said to be $a$ " coregular sequence on $A$ " if $\omega_{1}^{*}, \omega_{2}^{*}, \ldots, \omega_{s}^{*}$ is a regular sequence on $A^{*}$.

For the next result we use $\Omega_{i}$ to denote the sequence $\omega_{1}, \omega_{2}, \ldots, \omega_{i}$. By $0:{ }_{A} \Omega_{0}$ we mean $A$ itself and we use $\Omega$ as an alternative to $\Omega_{s}$. objects

Proposition 6. The sequence $\omega_{1}, \omega_{2}, \ldots, \omega_{s}$ is coregular on $A$ if and only if each of the

$$
\left(0:{ }_{A} \Omega_{i-1}\right) / \omega_{i}\left(0:{ }_{A} \Omega_{i-1}\right) \quad(1 \leqq i \leqq s)
$$

is $\Omega$-cotrivial.

Proof. We have

$$
\left(\Omega_{i-1}^{*} A^{*}:{ }_{A^{*}} \omega_{i}^{*}\right) / \Omega_{i-1}^{*} A^{*}=0: A_{A^{*} / \Omega_{i}^{*}-1 A^{*}} \omega_{i}^{*}
$$

and, by (4.4), the dual of this is

$$
\left(A^{*} / \Omega_{i-1}^{*} A^{*}\right)^{*} / \omega_{i}\left(A^{*} / \Omega_{i-1}^{*} A^{*}\right)^{*} .
$$

However, again by (4.4),

$$
A^{*} / \Omega_{i-1}^{*} A^{*}=\left(0: \Omega_{i-1}\right)^{*}
$$

Thus the dual of

$$
\left(\Omega_{i-1}^{*} A^{*}{ }_{A^{*}} \omega_{i}^{*}\right) / \Omega_{i-1}^{*} A^{*}
$$

is

$$
\left(0:{ }_{A} \Omega_{i-1}\right) / \omega_{i}\left(0:_{A} \Omega_{i-1}\right)
$$

and therefore the latter is $\Omega$-cotrivial precisely when the former is $\Omega^{*}$-trivial. The proposition follows. 
It is now necessary to observe that the effect of applying the duality $G: \mathfrak{A} \rightarrow \mathfrak{I}^{*}$ to the Koszul complex $K\left(\omega_{1}, \omega_{2}, \ldots, \omega_{s} \mid A\right)$ is to produce the complex

$$
K\left(\omega_{1}^{*},-\omega_{2}^{*}, \ldots,(-1)^{s-1} \omega_{s}^{*} \mid A^{*}\right) .
$$

This, however, requires some amplification.

Suppose that $0 \leqq \mu \leqq s$ and let $i_{1}, i_{2}, \ldots, i_{\mu}$ and $j_{1}, j_{2}, \ldots, j_{s-\mu}$ be sequences of integers satisfying $1 \leqq i_{1}<i_{2}<\ldots<i_{\mu} \leqq s$ and $1 \leqq j_{1}<j_{2}<\ldots<j_{s-\mu} \leqq s$. These two sequences will be said to be complementary if between them they contain all the integers $1,2, \ldots, s$. Consider $K_{\mu}\left(\omega_{1}, \omega_{2}, \ldots, \omega_{s} \mid A\right)$ and $K_{s-\mu}\left(\omega_{1}^{*},-\omega_{2}^{*}, \ldots,(-1)^{s-1} \omega_{s}^{*} \mid A^{*}\right)$. The former is the biproduct of $\left(\begin{array}{l}s \\ \mu\end{array}\right)$ terms $A_{i_{1} i_{2} \ldots i_{\mu}}$ whereas the latter is the biproduct of $\left(\begin{array}{l}s-\mu \\ s\end{array}\right)=\left(\begin{array}{l}s \\ \mu\end{array}\right)$ terms $\left(A^{*}\right)_{j_{1} j_{2}}$ Accordingly we may identify

$$
\left(K_{\mu}\left(\omega_{1}, \omega_{2}, \ldots, \omega_{s} \mid A\right)\right)^{*} \text { and } K_{s-\mu}\left(\omega_{1}^{*},-\omega_{2}^{*}, \ldots,(-1)^{s-1} \omega_{s}^{*} \mid A^{*}\right)
$$

in such a way that, when $i_{1}, i_{2}, \ldots, i_{\mu}$ and $j_{1}, j_{2}, \ldots, j_{s-\mu}$ are complementary, $\left(A_{i_{1} i_{2} \ldots i_{\mu}}\right)^{*}$ becomes identified with $\left(A^{*}\right)_{j_{1} j_{2} \ldots j_{s-\mu}}$. Assume this has been done. On applying the duality to the boundary morphism

$$
d_{\mu}: K_{\mu}\left(\omega_{1}, \omega_{2}, \ldots, \omega_{s} \mid A\right) \rightarrow K_{\mu-1}\left(\omega_{1}, \omega_{2}, \ldots, \omega_{s} \mid A\right)
$$

and making the identification described in (4.6), we obtain the $\mathfrak{I}^{*}$-morphism

$$
d_{\mu}^{*}: K_{s-\mu+1}\left(\omega_{1}^{*},-\omega_{2}^{*}, \ldots,(-1)^{s-1} \omega_{s}^{*} \mid A^{*}\right) \rightarrow K_{s-\mu}\left(\omega_{1}^{*},-\omega_{2}^{*}, \ldots,(-1)^{s-1} \omega_{s}^{*} \mid A^{*}\right) .
$$

A straightforward check then shows that this is none other than the boundary morphism of degree $s-\mu+1$ of the Koszul complex $K\left(\omega_{1}^{*},-\omega_{2}^{*}, \ldots,(-1)^{s-1} \omega_{s}^{*} \mid A^{*}\right)$. The details of this verification are left to the reader. It follows that

$$
\left(H_{\mu} K\left(\omega_{1}, \omega_{2}, \ldots, \omega_{s} \mid A\right)\right)^{*}=H_{s-\mu} K\left(\omega_{1}^{*},-\omega_{2}^{*}, \ldots,(-1)^{s-1} \omega_{s}^{*} \mid A^{*}\right) .
$$

LEMMA 12. With the above notation $H_{\mu} K\left(\omega_{1}, \omega_{2}, \ldots, \omega_{s} \mid A\right)=0$ if and only if

$$
H_{s-\mu} K\left(\omega_{1}^{*}, \omega_{2}^{*}, \ldots, \omega_{s}^{*} \mid A^{*}\right)=0 \text {. }
$$

This follows from (4.7) when taken in conjunction with (3.2).

We now have all the necessary machinery to enable us to dualise the main results of Section 3. In this way we obtain the theorems listed below.

THEOREM $1^{*}$. Let $A$ be an $\Omega$-conormal $\mathfrak{A}$-object, where $\Omega=\left\{\omega_{1}, \omega_{2}, \ldots, \omega_{s}\right\}$. If, for some integer $p \leqq s, H_{p} K\left(\omega_{1}, \omega_{2}, \ldots, \omega_{s} \mid A\right)=0$ then $H_{q} K\left(\omega_{1}, \omega_{2}, \ldots, \omega_{s} \mid A\right)=0$ for all $q \leqq p$.

THEOREM 2*. Let the $\mathfrak{A}$-object $A$ be $\Omega$-conormal, where $\Omega=\left\{\omega_{1}, \omega_{2}, \ldots, \omega_{s}\right\}$. Then the following statements are equivalent:

(a) $\omega_{1}, \omega_{2}, \ldots, \omega_{s}$ is a coregular sequence on $A$,

(b) $H_{s-1} K\left(\omega_{1}, \omega_{2}, \ldots, \omega_{s} \mid A\right)=0$. 
Theorem $3^{*}$. Suppose that $\Omega=\left\{\omega_{1}, \omega_{2}, \ldots, \omega_{s}\right\}$ and that $\left\{v_{1}, v_{2}, \ldots, v_{s}\right\}$ is a permutation of $\{1,2, \ldots, s\}$. If $\omega_{1}, \omega_{2}, \ldots, \omega_{s}$ is a coregular sequence on the $\Omega$-conormal $\mathfrak{U}$-object $A$ then $\omega_{v_{1}}, \omega_{v_{2}}, \ldots, \omega_{v_{s}}$ is also a coregular sequence on $A$.

THEOREM 4*. Suppose that $\Omega=\left\{\omega_{1}, \omega_{2}, \ldots, \omega_{s}\right\}$ and that $p_{1}, p_{2}, \ldots, p_{s}$ are positive integers. If $\omega_{1}, \omega_{2}, \ldots, \omega_{s}$ is a coregular sequence on the $\Omega$-conormal $\mathfrak{U}$-object $A$, then $\omega_{1}^{p_{1}}, \omega_{2}^{p_{2}}, \ldots, \omega_{s}^{p_{s}}$ is also a coregular sequence on $A$.

\section{REFERENCES}

1. M. Auslander and D. A. Buchsbaum, Codimension and multiplicity, Ann. of Math. 68 (1958), 625-657.

2. D. G. Northcott, Generalized $R$-sequences; to appear. 1968).

3. D. G. Northcott, Lessons on rings, modules and multiplicities (Cambridge University Press,

\section{THE UNIVERSITY}

SHEFFIELD 